\title{
El ushnu y la organización espacial astronómica en la sierra central del Chinchaysuyu
}

José Luis Pino M. ${ }^{1}$

\section{RESUMEN}

El ushnu inca tuvo gran importancia en la dominación ideológica en los wamani del Chinchaysuyu. Este artículo ofrece nuevas interpretaciones basadas principalmente en evidencias encontradas en Huánuco Pampa y otros tampus de la sierra central. Se propone que el ushnu en los Andes sólo tuvo funciones de recepción de ofrendas, especialmente líquidos, en ceremonias programadas de acuerdo a un calendario instituido, y que posteriormente los incas superponen funciones de observación astronómica. Se demuestra que estos tampus inca fueron planificados de acuerdo a la ubicación del ushnu y de determinados alineamientos astronómicos, basándose en un posible modelo de organización espacial radial astronómica.

Palabras claves: ushnu - Imperio Inca - Chinchaysuyu astronomía - organización del espacio.

\section{ABSTRACT}

The inca ushnu was an important element of ideological domination in the wamani of the Chinchaysuyu. This article offers new interpretations based on evidence found in Huánuco Pampa and other tampus of the central sierra. We propose that the Andean ushnu functioned only to receive offerings, fluids especially, in ceremonies that were scheduled according to an instituted calendar, and that the incas later superimposed astronomical observation functions. We demonstrate that these inca tampus were built in accordance to the localization of the ushnu and of specific astronomical alignments, possibly based on a radial model of astronomical spatial organization.

Key words: ushnu - Inca Empire - Chinchaysuyu astronomy - spatial organization.

Recibido: mayo 2004. Manuscrito revisado aceptado: marzo 2005.

1 Universidad Nacional Mayor de San Marcos. Av. Melgarejo 463, Santa Patricia - La Molina. Lima, PERU. Email: josepinomatos@peru.com
"Pacha Cuti Ynga dio orden muy mucha hazienda para sacrificar a las uacas y de las casas del so y tenplo de Curi Cancha; el trono y aciento de los Yngas llamado usno en cada wamani señaló" (Guamán Poma 1980 [1615]: 265 [267]).

\section{Introducción}

La sociedad inca en su política imperial utilizó muchas ideas desarrolladas por sociedades anteriores a ella, entre las cuales la del ushnu fue empleada como parte de la dominación ideológica en los territorios conquistados, sobre todo en la región del Chinchaysuyu con más monumentalidad. Nuestra investigación presenta hipótesis sobre el probable proceso de evolución del concepto "ushnu", su uso y posterior superposición de funciones por los incas, para después convertirse en un elemento importante en la planificación de los asentamientos de las capitales de provincia o wamani.

Si hablamos de planificación de asentamientos, hacemos referencia a la organización del espacio que debió desarrollar la sociedad inca, la cual no podía separar su concepción de tiempo de la de espacio, por lo tanto, ambos conceptos iban unidos y eran nombrados con una misma palabra que define conceptos polisemánticos: "Pacha" (espacio y tiempo). ${ }^{2}$ Asimismo, los incas trataron de representar en los wamanis o provincias, la forma de organización espacial que existió en su capital imperial, el Cusco, en algunos casos con muchos detalles representativos de su ideología, constituyendo "nuevos Cuscos", no de forma exacta, pero sí adaptando en muchos casos a situaciones particulares de los territorios conquistados.

2 "Sabemos que en quechua las nociones de espacio y tiempo son designadas por un mismo término, "pacha", que significa al mismo tiempo (siguiendo el contexto), la tierra, el mundo, etc., o la época, la estación, etc." (Wachtel 1973: 181). 
El área en la que se centra este estudio es la sierra central del Chinchaysuyu, principalmente los wamanis de Huánuco y Chinchaycocha, teniendo como capitales provinciales los tampus de Huánuco Pampa y Pumpu, respectivamente (Figura 1).

\section{Estudios sobre el ushnu}

Las referencias etnohistóricas sobre el ushnu han sido estudiadas por varios investigadores, quienes han planteado diversas interpretaciones sobre su naturaleza (Zuidema 1980; Matos 1986; Hyslop 1990; Meddens 1997). Sin embargo, cabe resaltar las referencias que dan ciertos documentos etnohistóricos que: a) indican que estaban construidos en plazas y dentro de la red vial incaica, así como las ofrendas de chicha que daban al sol en estos lugares (Albornoz 1967 [1582]: 24); b) enfatizan sobre determinadas ceremonias, dirigidas al sol, a las huacas, refiriéndose específicamente al Capac Hucha ${ }^{3}$ (Guamán Poma 1980 [1615]: 262 [264]) y, sobre todo, señalan que se trata de lugares de sacrificio y libación (Hernández Príncipe 1923: 63).

Entre las primeras investigaciones, Zuidema presenta una amplia y acuciosa información sobre el tema, desarrollando en sus investigaciones diversos planteamientos sobre la ideología inca, los cuales han servido a los posteriores investigadores como un marco referencial para entender el ushnu, sugiriendo que éste tiene un carácter de acceso al "mundo subterráneo" (Zuidema 1989: 408), asociando la acción de "chupar" o absorber las ofrendas líquidas en los rituales realizados. Pero, a la vez, menciona que el ushnu en Cusco fue un centro de observaciones astronómicas y que posiblemente influyó en el trazo del plano general de esa ciudad (Zuidema 1989: 453). Por su parte, Hyslop planteó una perspectiva a nivel imperial, describiendo al ushnu como plataformas construidas al centro o al lado de las plazas en los tampus. Habrían tenido un papel central principalmente en las actividades políticas, religiosas y militares, siendo un eje de la vida ceremonial inca, teniendo un rol más público en provincias, por lo que las plataformas fueron más grandes (Hyslop 1990).

3 La Capac Hucha fue posiblemente la más grande de las ceremonias realizadas en el Imperio inca. Ha sido descrita por Duviols (1976) y Zuidema (1989).
Las investigaciones a un nivel regional, realizadas por Matos en el tampu de Pumpu, revelan que:

“(...) el ushnu quizás fue el primer edificio construido en Pumpu, luego la plaza seguida de los barrios y sectores de la ciudad. Desde este punto se habría controlado y dirigido la construcción de toda el área" (Matos 1986: 59).

Matos (1986: 50) percibe que el ushnu "habría jugado un papel muy importante desde el comienzo de la planificación de la ciudad".

De este modo, realizando una revisión de la información publicada acerca del tema, y una constatación en el campo, pudimos darnos cuenta de ciertas recurrencias en los wamanis de Huánuco y Chinchaycocha, notando que el ushnu-además de sus características simbólicas inherentes y sus características físicas de plataformas receptoras de ofrendas- fue usado como punto primordial o eje para la planificación de los tampus, teniendo como base alineamientos astronómicos. Es decir, en términos conceptuales, los incas realizaron el ordenamiento del espacio en base al tiempo y viceversa, como unidad "Pacha" (Pino 2001 Ms-a).

La idea de ushnu y su relación con el Chinchaysuyu

Zuidema es enfático sobre el carácter ctónico que posee el ushnu. Asimismo, sobre su procedencia, él sospecha que la palabra ushnu no fue original del Cusco, pero los incas le dieron mucha importancia como concepto político, no relacionándolo con el centro (Cusco) sino con el norte, entendido como el Chinchaysuyu (Zuidema 1989: 413).

La información etnográfica, registrada por el trabajo de campo que realicé los años 1999 y $2000^{4}$ en la sierra central del Perú, me permitió proponer la posible "área nativa" del concepto ushnu, así como el probable significado primario del mismo. ${ }^{5} \mathrm{El}$ análisis del registro etnográfico y la toponimia, muestra que, entre las áreas de Tarma

4 Trabajo que tomó como referencia las áreas inmediatas por las que recorrió la ruta principal del Qhapaqñan del Chinchaysuyu que va al norte, entre los departamentos actuales de Cusco, Apurímac, Ayacucho, Huancavelica, Junín, Cerro de Pasco, Huánuco y Ancash (Pino 2001 Ms-b).

5 Entiéndase primario como: primitivo y, a la vez, principal y primero en orden. 


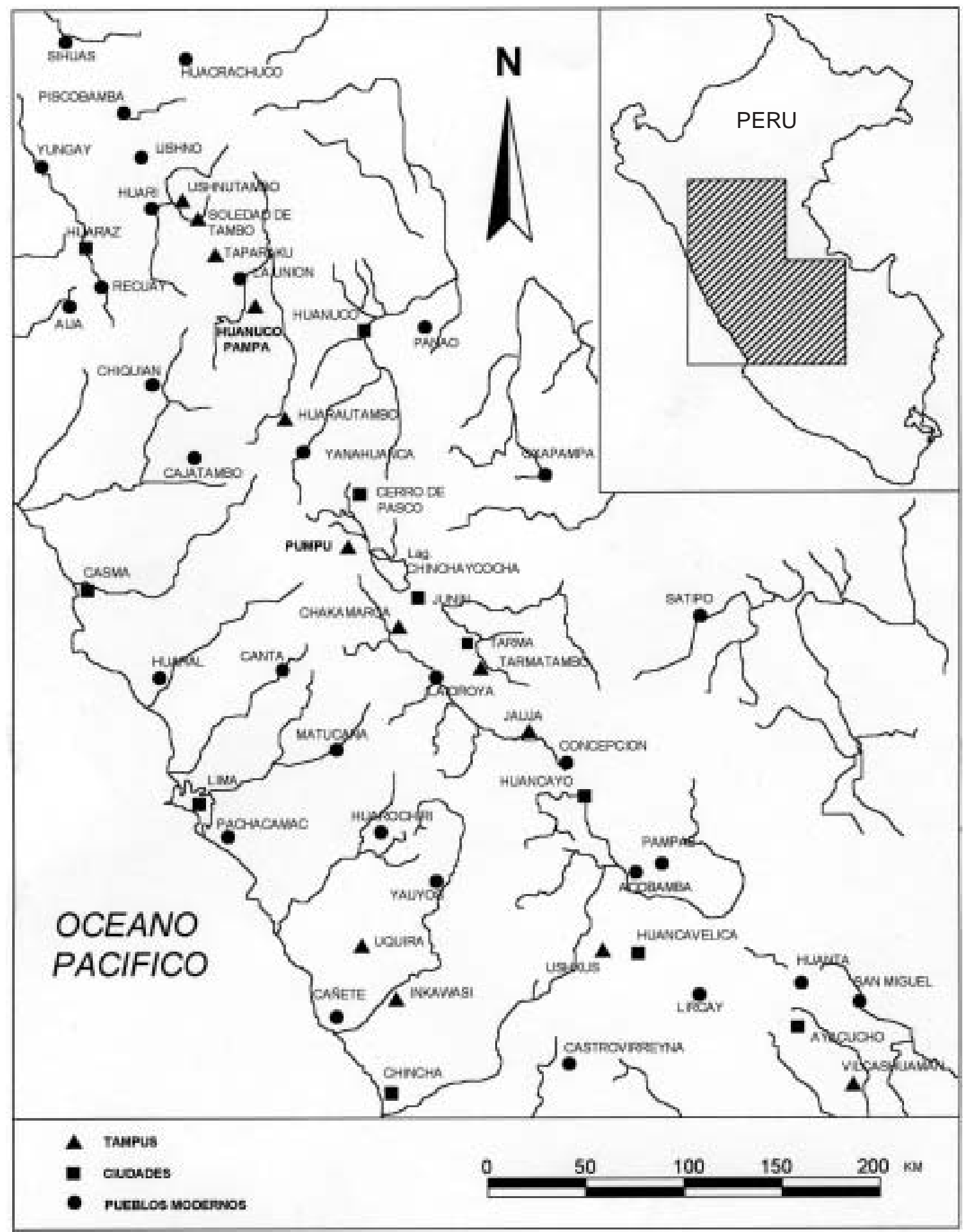

Figura 1. Región central del Chinchaysuyu. 
(Junín) hasta Sihuas (Ancash), pertenecientes a la región del Chinchaysuyu en la época inca, la denominación ushno aún es muy utilizada, ${ }^{6}$ y con diferentes connotaciones. Los significados más recurrentes a los que alude la población nativa en estas áreas son: huecos, subsuelo, ruinas, pircas (muros de piedra), lugares donde se encuentran los muertos, lugares donde están los "gentiles" (ancestros o población prehispánica), lugares peligrosos donde salen enfermedades, lugares donde se filtra el agua, lugares donde el agua es chupada, pozos, lugares donde hay mucho cascajo, lugares de puras piedras, y plataformas en las cimas de los cerros más altos. Resalta un área significativa ${ }^{7}$ como el Callejón de Conchucos, en la sierra del departamento de Ancash, cuya población en su mayoría es la que mejor define el concepto, empleando esta palabra para designar los lugares "donde hay mucho cascajo" y "donde hay pura piedra" (Pino 2001 Ms-b).

En el área entre los distritos de Chavín de Huantar y Huari (ambos en Ancash), el empleo del término ushno es muy claro y específico, pues la población nativa usa esta palabra para referirse al lugar compuesto de puras piedras o cascajo donde se filtra el agua. En esta área incluso se encuentran dos sitios, los cuales físicamente se adecuan al concepto mencionado: el primero es un poblado llamado Ushno, el cual se diferencia de los demás poblados existentes alrededor por asentarse en una zona de puro cascajo, junto a una quebrada por la que corre un riachuelo que atraviesa el poblado antes de llegar al río Huari. ${ }^{8}$ El segundo es un sitio arqueológico inca denominado Ushnotambo por los pobladores, por estar

6 Utilizando la palabra ushno entre las áreas de Sihuas y Chavín de Huantar (Ancash); ushnu en el área de Mollebamba (La Libertad); oshno en el área de Chavín de Pariarca (Huánuco); ushno entre las áreas de La Unión (Huánuco), Cerro de Pasco (Pasco), hasta Tarma (Junín); usno en las áreas de Huancavelica, Ayacucho y Apurímac (Pino 2001 Ms-b).

7 El área comprendida por los distritos de Chavín de Huantar, San Marcos, Huachis, Pomachaca y Huari (todos en la sierra de Ancash).

8 "El Caserío de Ushno: Este sitio ubicado a unos $10 \mathrm{~km} \mathrm{al}$ norte de Huari, es un caserío compuesto por unas siete u ocho casas, de población agricultora. Las características de este sitio son: a) ubicado en el cono de deyección de una quebrada lateral del río Huari; b) en la ruta del Camino Inca que va de Huaritambo al norte; c) existen chullpas pequeñas y bien conservadas; d) pero la característica principal es que está ubicado sobre una terraza de cascajo, o piedras angulosas de gran tamaño, estando sobre éstas otras ubicado al final de una acequia donde se pierde el agua de riego para llegar a un terreno muy pedregoso. ${ }^{9}$

Lo anterior nos lleva a plantear de forma preliminar, que ushnu es simplemente "el lugar compuesto de piedras donde se filtra el agua", y que éste fue posiblemente el concepto inicial. ${ }^{10}$ Estos lugares con características de ushnu tuvieron más factibilidad para la recepción de ofrendas líquidas, por lo que probablemente las poblaciones prehispánicas los utilizaron para realizar rituales donde se ofrendaban líquidos, principalmente chicha, constituyendo así "ushnu" un concepto ceremonial que posiblemente significaba "el lugar donde se ofrendaban líquidos, o lugar de libaciones".

\section{El ushnu en el desarrollo imperial inca}

El Cuzco, capital del Imperio, tuvo dos lugares de gran importancia donde se realizaban libaciones a las deidades, ambos considerados como "centro": el primero, de carácter más cerrado y sagrado fue el templo de Qorikancha y, el segundo, la plaza Haukaypata, con ushnu, de carácter más público y político (Hyslop 1990; Farrington 1998). Las descripciones en las crónicas sobre el ushnu

de menor tamaño. Y sobre este depósito de piedras han construido terrazas agrícolas, las cuales al ser regadas absorben rápidamente el agua, y ésta vuelve a salir por debajo de las piedras para dirigirse al riachuelo o al río Huari. Y al preguntar a la población sobre el significado de la palabra ushno, dan la descripción de chacras donde el agua se absorbe rápidamente por tener mucha piedra debajo, y hacen referencia y ponen como ejemplo este lugar" (Pino 2001 Ms-b).

9 "Ushnutambo: Sitio arqueológico ubicado al norte de Chavín de Huantar y al sur de Huari, en el tramo del Qhapaqñan que une los tampus incas de Soledad de Tambo y Huaritambo, asentamiento que está sobre una zona de mucho cascajo, y que la población de los distritos de Huachis y Pomachaca denomina así, por ser un lugar donde el agua se filtra por las piedras y ahí acaba la acequia, desconociendo dónde se va el agua de riego. Este sitio arqueológico está compuesto por dos estructuras rectangulares de albañilería sencilla, adyacentes al camino" (Pino 2001 Ms-b).

10 La información etnográfica recuperada en el área de Chavín de Huantar y Huari (a diferencia de las otras áreas muestreadas) sobre el significado de ushno, nos permite plantear la posible área nativa y el probable significado primario del mismo, debido a que en esta zona los informantes se refieren, aún en lengua nativa (quechua local), a ushno como "lugar compuesto de piedras donde se filtra el agua", como un lugar de fácil observación, y no a definiciones de conceptos ideológicos que pudieran haberse transformado a través del tiempo por las influencias coloniales y republicanas. 
del Haukaypata, son muy variadas, pero las informaciones estudiadas permiten saber que existió un monumento de piedra con un recipiente y su sistema de drenaje (Zuidema 1980; Hyslop 1990). El lugar de ofrendas líquidas del ushnu en Cusco tuvo el mismo sentido del concepto ceremonial propuesto; pero con una forma física mejor lograda, siendo no sólo un lugar de piedras donde filtre las ofrendas líquidas, sino un recipiente donde verter las ofrendas, con un sistema por donde drenarlo para evitar que se llene; creando de esta manera un mecanismo, para tener la idea de que la deidad a quien se ofrecen los líquidos está bebiendo o simule que está chupando.

Sin embargo, este artificio se vuelve complejo cuando construyen los ushnu en los lugares conquistados: adoptando la forma física de plataformas, que además de tener un lugar donde echar las ofrendas líquidas y su respectivo sistema de drenaje, presentan escalinatas y accesos para ingresar a la parte superior de las plataformas, como también asientos o tianas en la cima de éstas; elaborando de diversas formas el lugar donde se vertían los líquidos, siendo generalmente pozos de ofrenda o cocha, los cuales en su mayoría tenían al interior un relleno de piedras por donde se filtraba la ofrenda líquida (Matos 1986, 1994; Raffino et al. 1997; Pino 2001 Ms-b). ${ }^{11}$ Pero, además de volverse complejo el artificio, el ushnu en la época inca no sólo sería el lugar destinado a ofrendas líquidas, sino también se relacionaría a sacrificios de niños, animales y quema de tejidos y otras ofrendas (Hyslop 1990: 100), haciéndose más complejas las ceremonias y las formas de ofrendar.

Las características físicas de los ushnus en el Imperio inca han sido descritas en las investigaciones de Zuidema (1980), Hyslop (1990), y Raffino y colaboradores (1997). Particularmente, en la zona central del Chinchaysuyu, en Huánuco Pampa destacan los trabajos de Shea (1966) y Morris y Thompson (1970, 1985), en Taparaku las de Serrudo (2002) y en Pumpu y Chakamarca

11 En el registro que realicé al tampu de Soledad de Tambo, ubicado en la ruta del Qhapaqñan que cruza el distrito de Huachis al sur de la Provincia de Huari (Ancash), identifiqué el ushnu entre los terrenos agrícolas y, en la parte superior de la plataforma, éste aún conserva su pozo de ofrendas casi intacto, el cual está elaborado de fina cantería al estilo cusqueño y con corte almohadillado y, al fondo del pozo, se observa un relleno de piedras menudas o cascajo (Pino 2001 Ms-b). las de Matos (1986, 1994, 1995) y Matos y colaboradores (1996).

En los tampus de los wamanis de Huánuco y Chinchaycocha, los ushnus difieren en tamaño y forma, pero coinciden en la posición casi central dentro del asentamiento. Esto haría que las ceremonias efectuadas adquirieran un mayor carácter de centralidad, apoyando la idea del ushnu como "centro" propuesta por Hyslop. Por ello, en las provincias inca, el ushnu y las ceremonias realizadas adquieren un rol más público y obviamente más político (Hyslop 1990: 100). Así el ushnu se constituye en el centro de las principales ceremonias, cuya realización debía regirse por un calendario, el cual debía determinarse previamente de forma astronómica.

\section{Planificación, organización radial y alineamientos astronómicos}

Las crónicas refieren que en Cusco el ushnu, además de ser un lugar de ofrendas, fue un lugar para realizar observaciones astronómicas. Así lo demuestran las investigaciones de Zuidema (1980, 1981), quien menciona que: "El ushnu fue el centro arquitectónico de la plaza del Cusco y junto con el Templo del Sol influyó en el trazo del plano general de la ciudad" (Zuidema 1989: 453). Por ello es que se percibe la superposición de funciones en el ushnu con relación a la astronomía y al diseño de la Capital Imperial.

La organización del espacio que existió en Cusco estuvo basada en el sistema de ceques, que por medio de líneas radiales imaginarias que partían del Qorikancha, agrupaba los espacios sagrados dispuestos sobre las líneas, a la vez que ordenaba el espacio social (Wachtel 1973; Zuidema 1995). El centro del sistema ceque fue el Qorikancha, pero algunas huacas de los ceques sirvieron como referencia para observaciones astronómicas que se hicieron desde el ushnu (Zuidema 1974/76, 1981), estando varias huacas y ceques asociados a fenómenos astronómicos específicos (Zuidema 1974/ 76). Por lo tanto, parte de la organización de este sistema sería radial y astronómico.

En las sociedades andinas la astronomía cumplió un rol importante, especialmente para los incas, quienes no sólo consideraron al sol, sino también al centro de la vía láctea para orientarse de manera más exacta, tal como lo propone Urton: "los incas tomaron la salida de Alfa Crucis como se- 
ñal para las orientaciones del sur, incorporando así el 'centro' de la esfera celeste en el plan de la ciudad del Cusco, capital incaica y 'centro' de la esfera terrestre" (Urton 1985: 486). Este autor observó además que el sistema radial de ceques estaba dividido en cuadrantes o suyus mediante referentes astronómicos, de tal manera que: "En el sistema ceque, el primer ceque de tres de los cuatro suyus indicaba una dirección cardinal" (Urton 1985: 485) correspondientes al este, norte y oeste, que dividen así los suyus de la mitad norte; y para la división de suyus al sur, queda orientado un ceque hacia la salida de la Cruz del Sur, específicamente al azimut de la salida de Alfa Crucis (Urton 1985). Esta manera de dividir el espacio basándose en los astros ha sobrevivido a través de los siglos en las áreas que los incas conquistaron, pues en el área de Chinchaycocha la población nativa aún percibe el espacio que habita con una visión astronómica, tal como lo avala el registro etnográfico de Matos sobre los pastores de las punas de Junín:

"La geografía física es entendida desde la perspectiva solar. La orientación se basa, en el eje por donde nace y muere el sol, en cuyos extremos se ubican la yunca (selva) y la yunga (costa), este y oeste, respectivamente. Estos dos puntos tienen nombres, el Inti-ccespimunan (este) y el Intiseccaycunan (oeste). Muy cerca al punto donde declina el sol, reconocen el eje del desplazamiento del chaska-goillor (estrella del amanecer). Para visualizar el sur reconocen las estrellas de la "cruz del sur", y las llaman "Santa Cruz". Para el norte no tienen indicador claro. Algunos mencionan a chocce-chinchay" (Matos 1994: 48-49).

La presencia de ushnus monumentales y la organización radial en los asentamientos administrativos observados fuera del Cusco fue notable en la política imperial inca (Hyslop 1990), tratando de crear representaciones totales o parciales de la organización de la capital. En la región del Chinchaysuyu, la organización espacial donde se conjuga de manera clara el uso del ushnu y el sistema radial, queda manifiesta en el tampи de Incawasi en la costa central, y en el tampu de Huánuco Pampa en la sierra central: en ambos, el centro de la organización radial es un ushnu. ${ }^{12} \mathrm{Se}$ observa, por lo tanto, que la organización de los espacios que los incas están creando se basa en ejes o axis, que generalmente son centros simbólicos importantes, como en Cusco fue el Qorikan- cha y en provincias probablemente lo fueron los ushnus. Entonces en algunas capitales provinciales: "El ushnu fue el primer punto a ubicar en el espacio, y basándose en él, diseñaron el trazado de los asentamientos" (Pino 2001 Ms-a), tal como fue propuesto para el caso de Pumpu (Matos 1986). Esta idea concuerda con la propuesta de Hyslop (1990) respecto del ushnu como "centro" en los asentamientos provinciales, pero no sólo como centro ceremonial sino también como centro físico, de donde partían los alineamientos astronómicos que sirvieron para la organización espacial (Pino 2001 Ms-a). En este sentido, Hyslop (1985) ofrece más información sobre el uso del ushnu para el caso de Incawasi. ${ }^{13}$

Las evidencias sobre las principales orientaciones astronómicas usadas por los incas han sido presentadas por Zuidema (1981), Hyslop (1990), Ziolkowski y Sadowski (1992) y Bauer y Dearborn (1998). Pero aquellas relacionadas con la organización espacial y el ushnu en los tampus de la sierra central del Chinchaysuyu, provienen de Pumpu (Matos 1986, 1994) y Huanuco Pampa (Harth-Terre 1964; Morris y Thompson 1970; Morris 1987), donde se evidencia que la línea eje que divide el sitio en dos partes simétricas se asocia al equinoccio solar (Morris 1987). Sin embargo, Pumpu y Huánuco Pampa no sólo tuvieron las asociaciones astronómicas descritas. En efecto, revisando el trazado de ambos tampus encontramos más alineamientos ${ }^{14}$ con distribución radial asociados a diferentes fenómenos astronó-

12 Específicamente en el sector " $F$ " de Incawasi de Cañete, tal como lo propone Hyslop (1985), y con todo el asentamiento de Huánuco Pampa como lo propone Morris (1987).

13 Si bien Hyslop estudió el factor astronómico presente en diferentes asentamientos incas del Tawantinsuyu, desarrolló con más detalle este tema en el tampu de Incawasi.

14 Para nuestro estudio utilizamos el término "alineamiento astronómico" como la línea orientada hacia un fenómeno astronómico (para este caso, posiciones de orto, ocaso y zenit de los astros), desde y hacia referentes físicos tangibles (llámese punto de observación y marcadores de horizonte), estos alineamientos pueden estar compuestos por estructuras, conjuntos de estructuras, accesos principales, calles, caminos y horizontes naturales del paisaje local, especialmente cimas y laderas de cerros. Y definimos "observatorios" como las estructuras que guardan en su diseño interno características especiales para la observación de fenómenos astronómicos. Estas construcciones pueden presentar uno o varios alineamientos astronómicos formados por sus elementos internos (accesos, ventanas, nichos, corredores, entre otros). 
micos, que también se presentan en los tampus secundarios de Chakamarca (al sur de Pumpu) y Taparaku (al norte de Huanuco Pampa), ${ }^{15}$ todos relacionados a la organización espacial que toma al ushnu como eje. Esto incluso se observa en otros tampus del Chinchaysuyu como Tambo Colorado y Vilcaswaman.

Propongo que existió un "modelo de organización espacial radial astronómica" que usaron los incas en la planificación de algunos tambos del Chinchaysuyu. El modelo estaría basado en que ubicaban los principales elementos de los que estaría compuesto el tampu sobre los alineamientos asociados a fenómenos astronómicos predeterminados desde el ushnu, siendo los principales elementos: las estructuras, conjunto de estructuras, accesos principales, calles, caminos y puentes.

Las evidencias que presento se asocian a fenómenos astronómicos. Han sido ordenadas por grupos de elementos observados, codificados con un número y letra que indica el grupo, y siglas que indican el fenómeno astronómico asociado (Tabla 1). ${ }^{16}$

Este modelo estaría compuesto por líneas radiales que indican alineamientos astronómicos, las cuales parten del ushnu como centro. Las líneas ubicadas hacia el este indican la salida de los astros, y hacia el oeste indican la puesta de los mismos (Figura 2).

Entre los elementos arquitectónicos destacan: los accesos a las "canchas simétricas proporcionales"; ${ }^{17}$ los sectores llamados incawasi o residen-

15 La información obtenida en Chakamarca y Taparaku es preliminar y se encuentra en proceso de investigación. La información básica y necesaria para plantear nuestras hipótesis se presenta en la Tabla 1.

16 Todos los alineamientos de fenómenos astronómicos presentados como evidencia parten del ushnu y se dirigen a los elementos arquitectónicos descritos, tal como se propone en la Figura 2. Los datos presentados fueron recogidos por el autor en trabajos de campo. La información existente para el sitio de Pumpu se tomó de Matos $(1986,1994)$ y Hyslop (1990); para Chakamarca de Matos (1994), Matos y colaboradores (1996) y Pino (2001 Ms-a); para Huánuco Pampa de Harth-Terre (1964), Morris y Thompson (1985), Morris (1987) y Pino (2001 Ms-a, 2003 Ms); para Taparaku de Serrudo (2002) y para otros tampus lejanos de la sierra central, de Gasparini y Margolies (1977) y Hyslop (1990).

17 Se trata de una cancha de planta cuadrangular, compuesta por un par de recintos de medidas iguales en cada uno de

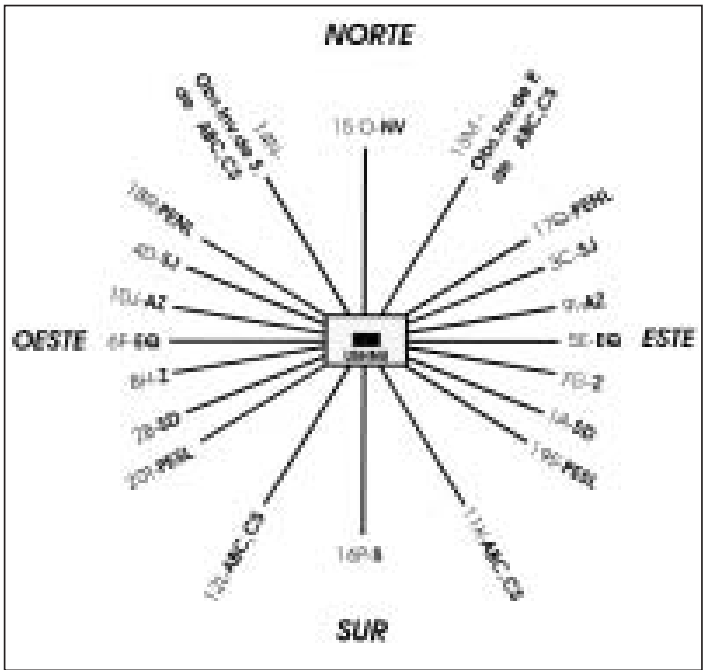

Figura 2. Modelo de organización espacial radial astronómica en el Chinchaysuyu.

cias de la élite administrativa; los aqllawasi, que generalmente están asociados a ejes direccionales relacionados con la Cruz del Sur, Alfa y Beta Centauri; ${ }^{18}$ esquinas de los aqllawasi en una dirección muy cercana al norte verdadero $;{ }^{19}$ los baños o fuentes de agua, muy bien construidos con piedra labrada al estilo cuzqueño ${ }^{20}$ asimismo, un grupo especial lo conforma el ushnu de Huánuco Pampa, pues además de servir de centro desde donde parten las líneas radiales astronómicas, tiene 10 tianas y dos accesos, por donde cruzan todas las direcciones arriba mencionadas, siempre pasando por el centro físico de la estructura que es el ushnu en sí mismo.

El modelo está dividido por las cuatro direcciones cardinales básicas en cuatro cuadrantes (NE, SE, NO, SO), cada uno con cuatro direcciones astronómicas, teniendo en total 20 direcciones utilizadas para la planificación en los tampus del Chinchaysuyu. Estas orientaciones astronómicas

sus cuatro lados, y podría ser la forma del conjunto principal del Qorikancha, tal como lo propuso M. Chávez (1970).

18 Los asentamientos Wari de Azangaro y Pikillacta tienen este alineamiento como eje del sitio.

19 NV, Norte Verdadero: La dirección debió estar determinada para su mayor exactitud por las observaciones de salida y puesta de ABC,CS, especialmente de Alfa Crucis, y las proyecciones inversas respectivas de estas observaciones señaladas.

20 Los baños incas estuvieron asociados con la luna llena y la luna nueva (Zuidema 1974/76: 227; Guamán Poma 1980 [1615]: 332 [334]). 
1A-SD: Salida del sol en el solsticio de diciembre: Acceso de la "cancha simétrica proporcional" del sector IIC de Huánuco Pampa; calle sureste de Pumpu.

2B-SD: Puesta del sol en el solsticio de diciembre: Lado sur de estructura larga junto a la plaza en el sector IVA en Huánuco Pampa; lado sur de la cancha trapezoidal del sector suroeste de Chakamarca.

3C-SJ: Salida del sol en el solsticio de junio: Acceso a la cancha del sector IIIA en Huánuco Pampa.

4D-SJ: Puesta del sol en el solsticio de junio: Acceso a la zona IVB de Huánuco Pampa.

5E-EQ: Salida del sol en el equinoccio: "cancha simétrica proporcional" Ad-9 en Pumpu; el sector del Incawasi o sector ceremonial IIB en Huánuco Pampa por donde cruza un sistema de pórticos con felinos tallados; tianas centrales de los lados este y oeste del ushnu de Huánuco Pampa; Piedra de los Sacrificios de Vilcaswaman.

6F-EQ: Puesta del sol en el equinoccio: Calle oeste de Huánuco Pampa; tianas centrales de los lados este y oeste del ushnu de Huánuco Pampa.

7G-Z: Salida del sol en su pasaje por el zenit: Cancha Ad-11 o sector del Incawasi en Pumpu; especialmente en posición casi exacta con el zenit está el baño de piedra ubicado en Pumpu; tiana sur del lado este del ushnu de Huánuco Pampa.

8H-Z: Puesta del sol en su pasaje por el zenit: Tiana sur del lado oeste del ushnu de Huánuco Pampa.

9I-AZ: Salida del sol en su pasaje por el antizenit: Cancha Ad-8 en Pumpu; tiana norte al lado este del ushnu de Huánuco Pampa.

10J-AZ: Puesta del sol en su pasaje por el antizenit: Tiana norte del lado oeste del ushnu en Huánuco Pampa.

11K-ABC,CS: Salida de Alfa, Beta Centauri y la Cruz del Sur: Cancha AD-16 en Pumpu; acceso sureste del ushnu de Huánuco Pampa, donde se encuentran dos pares de felinos tallados en cada lado del acceso; sector sur de Tambo Colorado; baño de piedra en el sector sur de Tambo Colorado.

12L-ABC,CS: Puesta de Alfa, Beta Centauri y la Cruz del Sur: Acceso suroeste del ushnu de Huánuco Pampa donde se encuentran dos pares de felinos tallados en cada lado del acceso; estructura sobre la plataforma de media luna en Pumpu.

13M: Observación inversa de puesta de ABC,CS: Esquina suroeste del aqllawasi de Pumpu; tiana extremo este del lado norte del ushnu de Huánuco Pampa; acceso principal al sector del incawasi en Tambo Colorado.

14N: Observación inversa de salida de ABC,CS: Tiana extremo oeste del lado norte del ushnu de Huánuco Pampa.

15 O-NV: Norte verdadero: Esquina suroeste del aqlawasi de Huánuco Pampa; esquina suroeste del aqllawasi de Taparaku. La determinación de esta dirección debió implicar mayor grado de precisión, por lo que la evidencia indica que está dirigido directamente a esquinas.

16P-S: Sur: Calle sur de Huánuco Pampa; calle sur de Pumpu.

17Q-S.PENL: Salida de la luna en su posición extrema norte: Estructuras gemelas de sillar en Taparaku; baño de piedra en Taparaku.

18R-P.PENL: Puesta de la luna en su posición extrema norte: Puente inca en Chakamarca; a través de incamesanan, que son dos plataformas separadas por donde pasa el camino en la salida noroeste de Huánuco Pampa.

19S-S.PESL: Salida de la luna en su posición extrema sur: Tiana central del lado este desde la tiana del extremo oeste del lado norte del ushnu de Huánuco Pampa.

20T-P.PESL: Puesta de la luna en su posición extrema sur: Tiana central del lado oeste desde la tiana del extremo este del lado norte del ushnu de Huánuco Pampa.

Tabla 1. Grupos de elementos arquitectónicos asociados a fenómenos astronómicos en los tampus del Chinchaysuyu.

usadas en el modelo propuesto tendrían una doble finalidad: organizar el espacio en los asentamientos incas y, a la vez, organizar el tiempo cons-

21 Información detallada sobre las actividades relacionadas a estos fenómenos astronómicos y reconstrucción de calendarios prehispánicos andinos se encuentran en el trabajo de Ziolkowski y Sadowski (1992), en el cual recopilan información proporcionada por diferentes cronistas y mencionan los posibles nombres de los meses del calendario tituyendo calendarios especialmente los asociados a solsticios, equinoccios y pasajes del sol por el

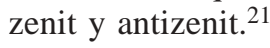

inca con sus fiestas y ceremonias (1992: 101-107). De la misma manera, Bauer y Dearborn presentan una lista de los meses incaicos a partir de la información recopilada de seis cronistas (1998: 48-49) y también D'Altroy (2003: 152-153) presenta de manera muy resumida un cuadro al respecto. 


\section{Huánuco Pampa, como mejor ejemplo de organización astronómica planificada}

Estudios de astronomía inca en Huánuco Pampa

Existen muy pocos estudios sobre astronomía inca para las áreas provinciales del Tawantinsuyu, sin embargo, la evidencia arqueológica presenta un mayor potencial en los tampus con carácter de centros administrativos en las capitales de los wamanis o provincias (Hyslop 1985, 1990; Matos 1986, 1994; Ziolkowski y Sadowski 1992). Huánuco Pampa, ubicado en la sierra central del Chinchaysuyu, a 3700 m.snm, posee una relación con los equinoccios por tener un eje este-oeste que cruza el sitio e influye en la organización espacial del tampu (Harth Terre 1964; Morris y Thompson 1970, 1985; Morris 1987).

Nuestra última investigación realizada en Huánuco Pampa $^{22}$ entrega nuevas evidencias de alineamientos y ofrece propuestas sobre observaciones astronómicas que realizaron los incas en este tampu, principios de organización espacial basadas en orientaciones astronómicas, así como la utilización específica de algunas estructuras como observatorios. Estas últimas son la plataforma ushnu y una estructura conocida en el lugar como el incawasi. El ushnu presenta además en Huánuco Pampa características de un observatorio astronómico complejo, que funcionaba mediante un sistema de asientos y accesos; y el incawasi (estructura de composición dual y cuatripartita) presenta en este caso un diseño especial para la observación de fenómenos astronómicos, que funciona por efectos de luz y sombra entre sus componentes

\footnotetext{
22 El objetivo principal de la investigación fue detectar alineamientos asociados a fenómenos astronómicos significativos o de especial importancia para la sociedad inca. Se utilizó para estos fines la información, planos y sectorización publicada por Morris y Thompson $(1970,1985)$ y Shea (1966), luego se elaboraron planos de mayor precisión de dos estructuras: el ushnu en el sector central y el incawasi en el sector oriental. Asimismo, para la determinación del norte verdadero y el registro de los alineamientos, usamos un teodolito Kern DKM2-A con precisión de un segundo de arco; el norte verdadero se obtuvo mediante observaciones solares, y se corrigió en base a las posiciones verdaderas del sol obtenidas con el programa HORIZONS Ephemerides Generator de la NASA y el Jet Propulsion Laboratory. Las correcciones de refracción atmosférica y paralaje fueron hechas utilizando las fórmulas y constantes publicadas por Meeus (2000a: 105,279).
}

internos (como accesos y nichos), utilizados para una mayor precisión en la determinación de ciertas fechas. La información arqueológica aquí presentada respalda ciertas propuestas sobre la astronomía inca, difíciles de comprobar en Cusco debido a la destrucción y/o ausencia de evidencia material. ${ }^{23}$

Los fenómenos astronómicos asociados a los alineamientos registrados en Huánuco Pampa son los que se nombran a continuación, simbolizados con las siglas entre paréntesis:

1) Salida del sol en el solsticio de diciembre: (SSSD)

2) Puesta del sol en el solsticio de diciembre: (PSSD)

3) Salida del sol en el solsticio de junio: (SSSJ)

4) Puesta del sol en el solsticio de junio: (PSSJ)

5) Salida del sol en el equinoccio: (SSEQ)

6) Puesta del sol en el equinoccio: (PSEQ)

7) Salida del sol el día de su paso por el zenit: (SSPZ)

8) Puesta del sol el día de su paso por el zenit: (PSPZ)

9) Salida del sol el día de su paso por el antizenit: (SSPAZ)

10) Puesta del sol el día de su paso por el antizenit: (PSPAZ)

11) Salida de Alfa, Beta Centaurus y la Cruz del Sur: (S-ABC,CS)

12) Puesta de Alfa, Beta Centaurus y la Cruz del Sur: (P-ABC,CS)

13) Salida de la luna en su posición extrema norte: (SLPEN)

14) Puesta de la luna en su posición extrema norte: (PLPEN)

15) Salida de la luna en su posición extrema sur: (SLPES)

16) Puesta de la luna en su posición extrema sur: (PLPES)

23 Zuidema en conjunto con Aveni realizaron estudios sobre astronomía inca durante varias temporadas en Cusco y alrededores, presentando propuestas sobre los lugares de observación, ubicación de los marcadores de horizonte y direcciones de los alineamientos, los cuales se asociarían a solsticios, pasos del sol y la luna por el zenit y antizenit, como también a la constelación de Las Pléyades (Zuidema 1981, 1983; Aveni 2001). Entre estas propuestas destaca la hipótesis sobre la importancia que tuvieron en la sociedad inca las fechas del paso del sol por el zenit y antizenit (Zuidema 1981: 322-324), idea que generó debate, al no existir evidencias físicas (de observatorios y marcadores de horizonte) relacionadas con dichos fenómenos (Bauer y Dearborn 1998: 120-121). 
Diseño del espacio en base alineamientos:

Evidencias en Huánuco Pampa

Los alineamientos astronómicos serían de dos tipos: a) a nivel de asentamiento, y b) a nivel de estructuras. Los primeros partían del centro del sitio, específicamente de la plataforma conocida como ushnu situada en medio de la plaza. Estos alineamientos estarían asociados a solsticios, equinoccios y posiciones extremas de la luna, y están orientados a elementos específicos de la arquitectura, especialmente a accesos y esquinas de los conjuntos de estructuras, como también a calles principales (Figura 3). Los alineamientos hacia el este se dirigen hacia los siguientes fenómenos astronómicos:

- SLPEN, por el lado norte del sector IIA.

- SSSJ, a través del acceso principal del sector IIA.

- SSEQ, a través de las cuatro portadas que cruzan el sector IIB, en dirección a un lugar llamado
Inkañan, ubicado en los cerros que sirven de horizonte al este.

- SSSD, a través del acceso principal de la cancha ortogonal del sector IIC.

- SLPES, por la esquina suroeste de la cancha ortogonal del sector IIC.

Y hacia el lado oeste se dirigen a:

- PLPEN, a través de la calle noroeste, y entre las dos plataformas conocidas como incamesanan, ubicadas a la salida del tampu, en el camino hacia el norte con dirección al tampu de Taparaku. - PSSJ, a través del acceso principal hacia el sector IVB.

- PSEQ, a través de la calle este.

- PSSD, por el lado sur de la estructura sur del sector IVA ubicada frente a la plaza.

- PLPES, a través de la calle suroeste.

Existen además orientaciones hacia el sur, que se dirigen hacia la calle sur, y hacia el norte, que se

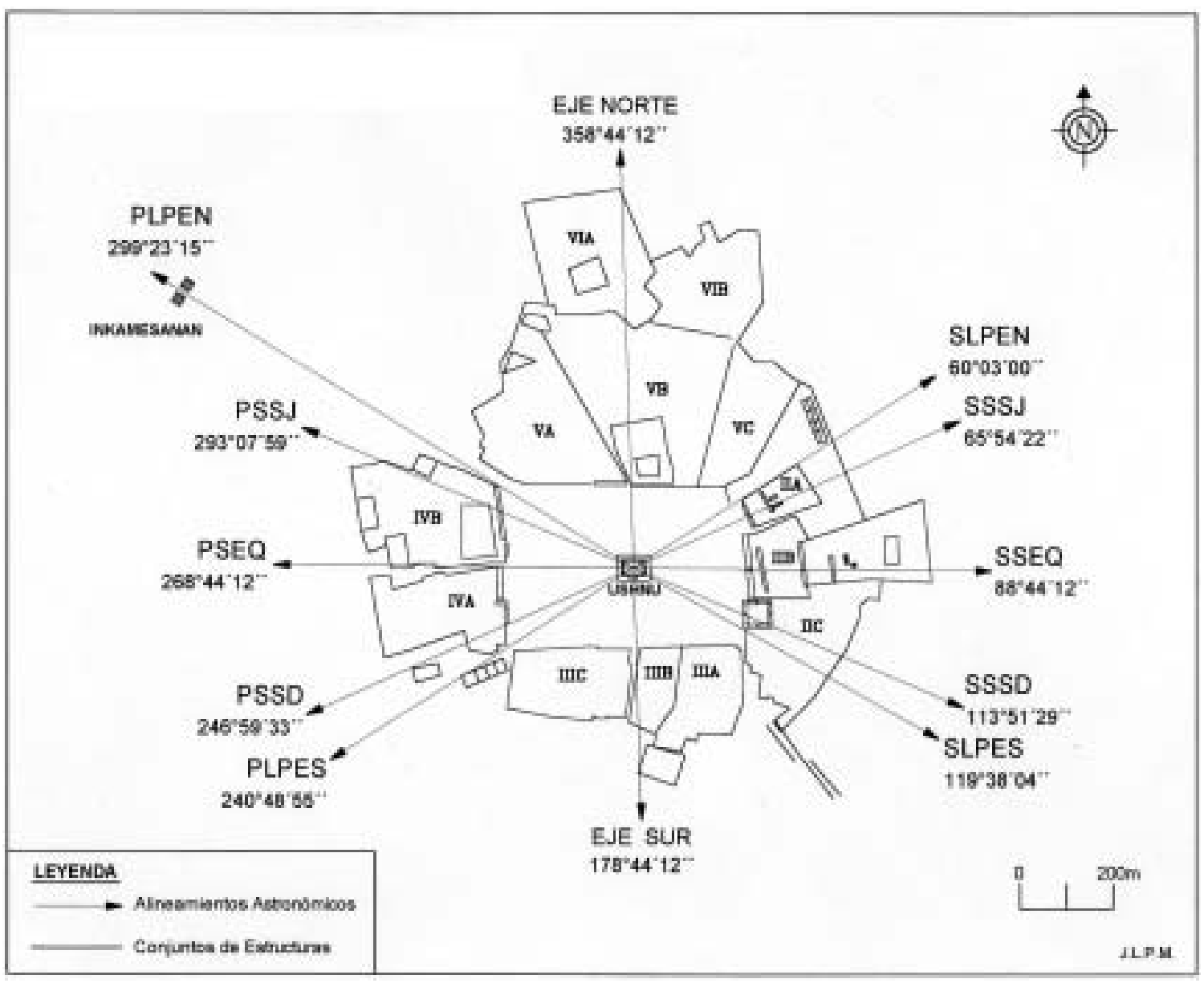

Figura 3. Alineamientos astronómicos en el tampu inca de Huánuco Pampa. 
dirigen hacia la esquina suroeste del aqllawasi. Estas ultimas orientaciones posiblemente las determinaron tomando la mitad de arco (la bisectriz) del ángulo formado por los alineamientos de $\mathrm{S}$ $\mathrm{ABC}, \mathrm{CS}$ y $\mathrm{P}-\mathrm{ABC}, \mathrm{CS}$, que trazaran desde el ushnu, debido a que estos alineamientos estelares determinan una mayor precisión y son de más fácil observación.

En segundo lugar, los alineamientos a nivel de estructuras se evidencian principalmente en dos de ellas: el ushnu e incawasi.

El ushnu (09 52' 39" Lat. Sur / 076 49'00" Long. Oeste, $3666 \mathrm{~m} . \mathrm{snm})$, situado en el sector central del tampu, es una gran plataforma rectangular de aproximadamente $32 \mathrm{~m}$ x $48 \mathrm{~m}$ de planta y $4 \mathrm{~m}$ de altura, construida en piedra labrada al estilo cusqueño, a la cual se accede por una escalinata y dos accesos (con medidas promedio de 3 $\mathrm{m}$ de largo y $1.40 \mathrm{~m}$ de ancho) en su lado sur. La parte superior está compuesta por muros a manera de parapetos (de $1.30 \mathrm{~m}$ de altura) y por 10 nichos que miran hacia el interior de la plataforma, conocidos como tianas por usarse como asientos. Estos nichos (de forma rectangular, de $2 \mathrm{~m}$ de largo, $0.80 \mathrm{~m}$ de altura y $0.50 \mathrm{~m}$ de profundidad) han sido edificados en el lado interior de los muros norte, este y oeste (Figura 4). Las tianas o asientos de piedra y los accesos se utilizaron para la conformación de los alineamientos, estos se formarían al dirigirse la orientación de una tiana hacia la otras opuestas (algunas perpendicularmen- te y otras de manera diagonal), y la mayoría atravesaría la parte central de la estructura (Figura 5). Estos alineamientos se dirigen hacia los fenómenos de SSSD, PSSD, SSSJ, PSSJ, SSEQ, PSEQ, SSPZ, PSPZ, SSPAZ, PSPAZ, S-ABC,CS, PABC,CS, SLPEN, PLPEN, SLPES y PLPES (Tabla 2). Lo anterior quiere decir que las personas encargadas de las observaciones se situaron en las tianas, orientándose hacia los lados opuestos con dirección a determinadas tianas, opuestas y accesos, con el objetivo de ubicar puntos específicos en el horizonte natural del paisaje que servirían en la observación de los fenómenos astronómicos.

La estructura llamada incawasi $\left(09^{\circ} 52^{\prime} 31^{\prime}\right.$ ' Lat. Sur / $076^{\circ} 48^{\prime} 43$ " Long. Oeste, $3644 \mathrm{~m} . \mathrm{snm}$ ) se ubica en el Sector II-B. Es una estructura doble, de planta rectangular de $9 \mathrm{~m} \times 23 \mathrm{~m}$, compuesta por dos partes iguales (norte y sur) divididas por un corredor central que atraviesa en un sentido suroeste-noreste. Cada parte de la estructura está dividida a su vez en dos recintos por un muro medianero (Figura 6) con accesos de forma trapezoidal ubicados hacia los lados este y oeste (con medidas promedio de $1.20 \mathrm{~m}$ de base, 2.35 $\mathrm{m}$ de altura y $1.05 \mathrm{~m}$ de ancho en el umbral). En el interior de los muros de cada recinto se encuentran 13 nichos trapezoidales (cada nicho con un promedio de medidas de $0.5 \mathrm{~m}$ de base, por $0.5 \mathrm{~m}$ de altura y $0.35 \mathrm{~m}$ de profundidad), a una altura de $1.90 \mathrm{~m}$ desde el nivel del suelo. Los nichos y los accesos principales de los recintos

\begin{tabular}{|c|c|c|c|c|}
\hline \multicolumn{2}{|c|}{ Alineamiento } & \multirow[t]{2}{*}{ Azimut } & \multirow{2}{*}{$\begin{array}{l}\text { Elevación } \\
\text { Horizonte }\end{array}$} & \multirow{2}{*}{$\begin{array}{c}\text { Fenómeno } \\
\text { asociado }\end{array}$} \\
\hline De: & A: & & & \\
\hline $\mathrm{TN} 2$ & TE1 & $114^{\circ} 27^{\prime} 50^{\prime \prime}$ & $02^{\circ} 25^{\prime} 40^{\prime \prime}$ & SSSD \\
\hline TN3 & TO1 & $245^{\circ} 49^{\prime} 20^{\prime \prime}$ & $05^{\circ} 18^{\prime} 52^{\prime \prime}$ & PSSD \\
\hline TW1 & TN3 & $65^{\circ} 49^{\prime} 20^{\prime \prime}$ & $03^{\circ} 07^{\prime} 20^{\prime \prime}$ & SSSJ \\
\hline TE1 & $\mathrm{TN} 2$ & $294^{\circ} 27^{\prime} 59^{\prime \prime}$ & $03^{\circ} 04^{\prime} 09^{\prime \prime}$ & PSSJ \\
\hline $\mathrm{TO} 2$ & TE2 & $88^{\circ} 44^{\prime} 12^{\prime \prime}$ & $02^{\circ} 45^{\prime} 25^{\prime \prime}$ & SSEQ \\
\hline TE2 & $\mathrm{TO} 2$ & $268^{\circ} 44^{\prime} 12^{\prime \prime}$ & $04^{\circ} 07^{\prime} 53^{\prime \prime}$ & PSQE \\
\hline TO1 & TE3 & $99^{\circ} 35^{\prime} 19^{\prime \prime}$ & $02^{\circ} 17^{\prime} 10^{\prime \prime}$ & SSPZ \\
\hline TE1 & TO3 & $260^{\circ} 06^{\prime} 50^{\prime \prime}$ & $02^{\circ} 48^{\prime} 17^{\prime \prime}$ & PSPZ \\
\hline TO3 & TE1 & $80^{\circ} 06^{\prime} 50^{\prime \prime}$ & $02^{\circ} 53^{\prime} 45^{\prime \prime}$ & SSPAZ \\
\hline TE3 & TO1 & $279^{\circ} 35^{\prime} 20^{\prime \prime}$ & $04^{\circ} 51^{\prime} 37^{\prime \prime}$ & PSPAZ \\
\hline $\mathrm{TN} 1$ & $\mathrm{AS} 2$ & $154^{\circ} 00^{\prime} 34^{\prime \prime}$ & $04^{\circ} 02^{\prime} 50^{\prime \prime}$ & S-ABC,CS \\
\hline TN4 & AS 1 & $203^{\circ} 22^{\prime} 00^{\prime \prime}$ & $08^{\circ} 22^{\prime} 02^{\prime \prime}$ & P-ABC,CS \\
\hline TO2 & TN4 & $60^{\circ} 40^{\prime} 29^{\prime \prime}$ & $03^{\circ} 18^{\prime} 20^{\prime \prime}$ & SLPEN \\
\hline TE1 & TN3 & $299^{\circ} 09^{\prime} 10^{\prime \prime}$ & $03^{\circ} 05^{\prime} 10^{\prime \prime}$ & PLPEN \\
\hline TN3 & TE1 & $119^{\circ} 09^{\prime} 10^{\prime \prime}$ & $02^{\circ} 24^{\prime} 54^{\prime \prime}$ & SLPES \\
\hline TN4 & TO2 & $240^{\circ} 40^{\prime} 29^{\prime \prime}$ & $07^{\circ} 46^{\prime} 04^{\prime \prime}$ & PLPES \\
\hline
\end{tabular}

Tabla 2. Alineamientos astronómicos registrados en el ushnu de Huánuco Pampa. 
JOSE LUIS PINO M.

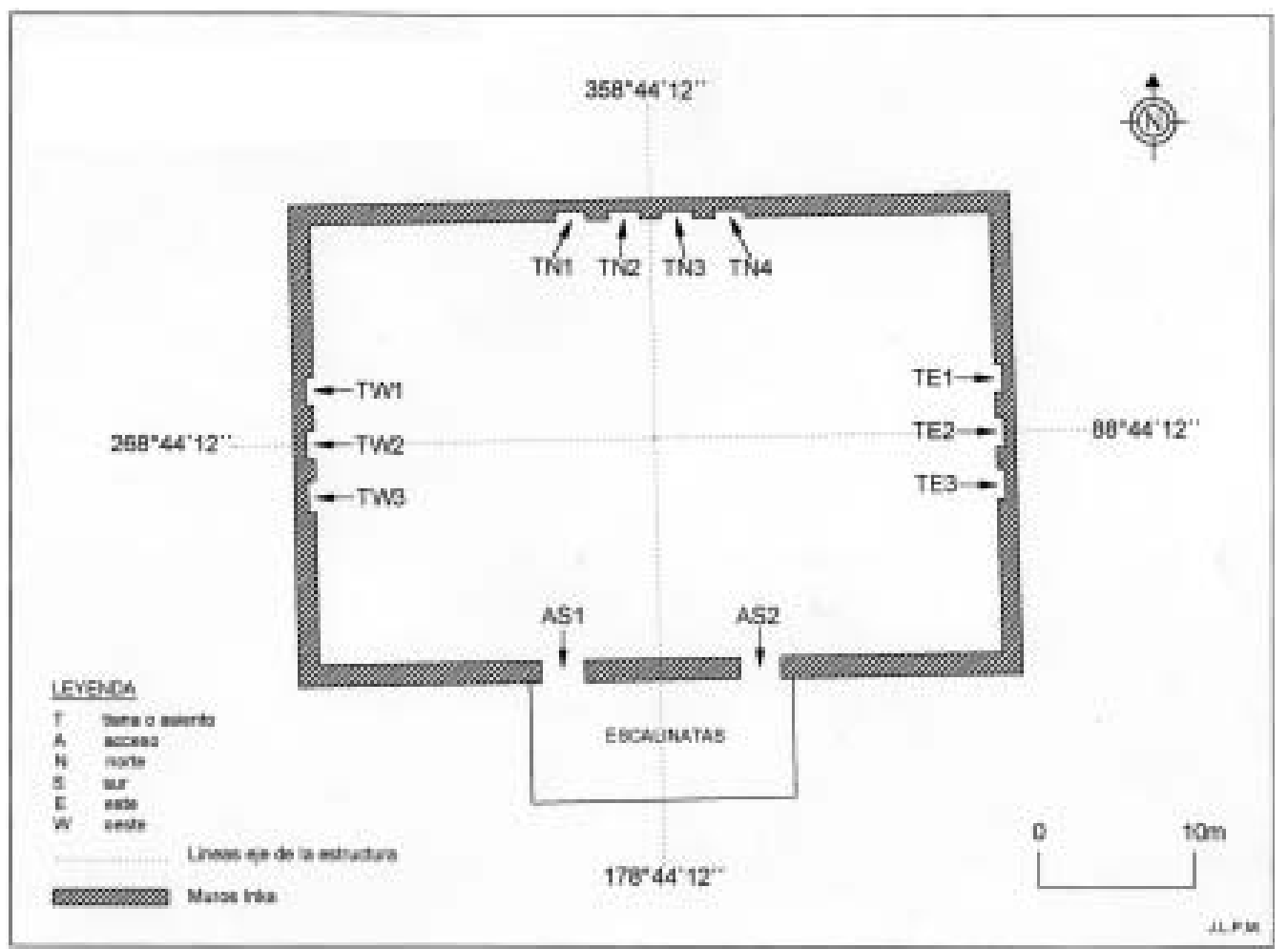

Figura 4. El ushnu inca de Huánuco Pampa, orientación y elementos internos.

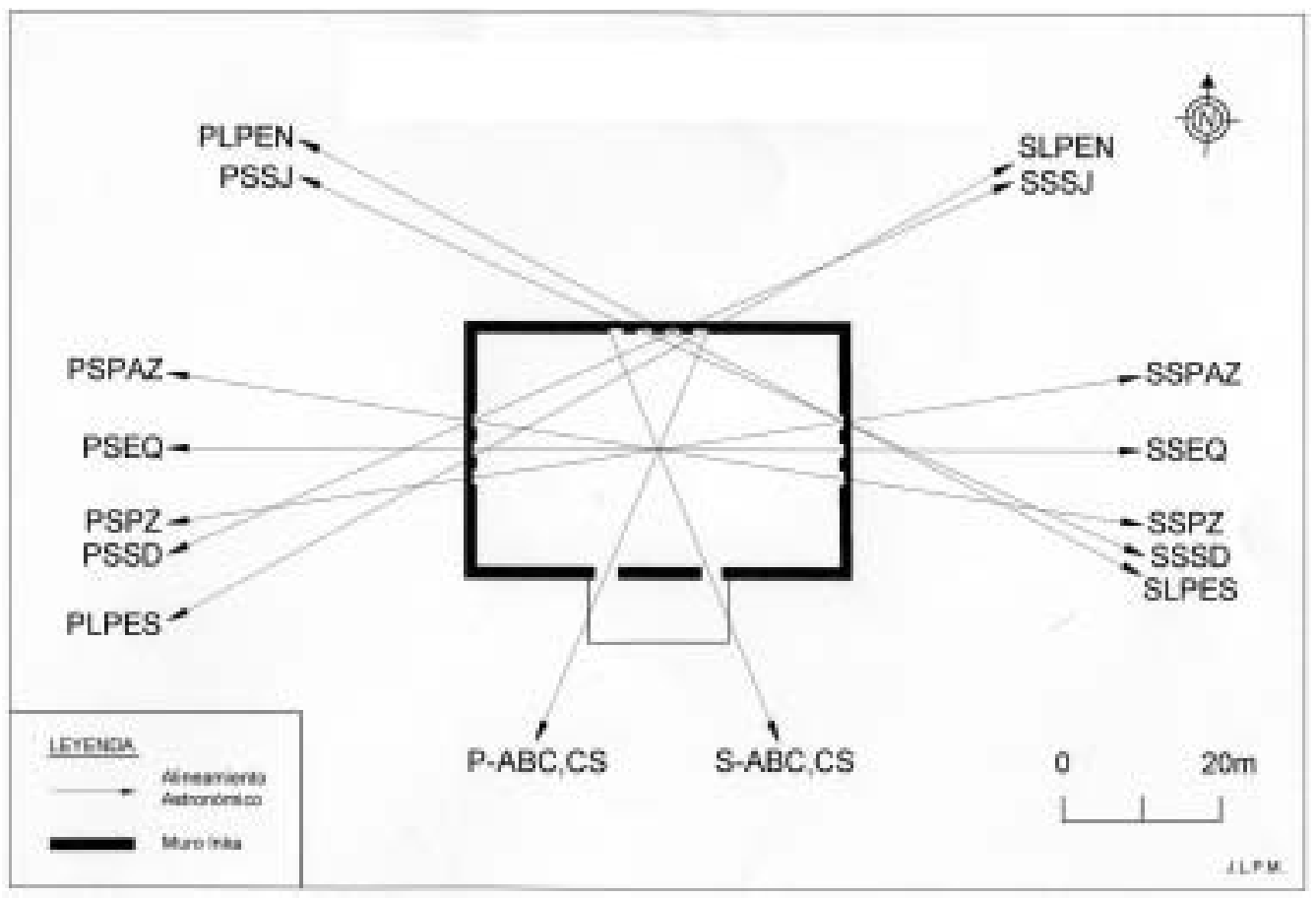

Figura 5. Alineamientos astronómicos en el ushnu, sector central de Huánuco Pampa. 


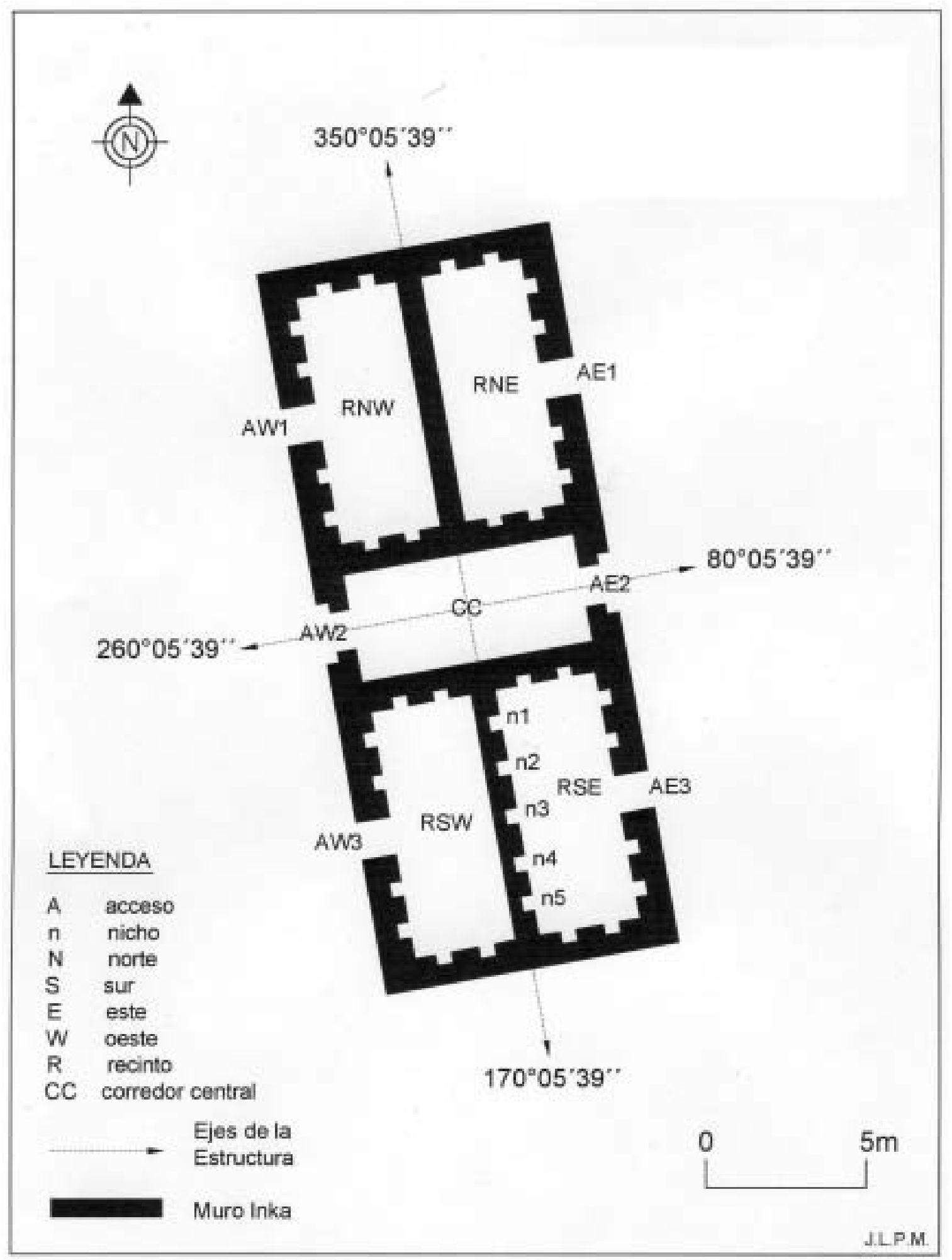

Figura 6. Estructura incawasi de Huánuco Pampa, orientación y elementos internos. 
sirven para la conformación de los alineamientos, dirigiéndose desde partes específicas de los nichos hacia el centro y partes específicas de los accesos. En el corredor central se utilizaron los centros de los accesos y las esquinas exteriores de los mismos. Los alineamientos se dirigen hacia los fenómenos de SSPAZ, SSPZ, PSPZ, SSEQ, PSEQ, SSSJ, SSSD, SLPEN y SLPES (Figura 7). La evidencia sobre alineamientos en los recintos se obtuvo del Recinto Sureste, por estar en mejores condiciones de conservación. Para llevar a cabo las observaciones astronómicas, las personas encargadas tuvieron que situarse al pie de los nichos o de partes específicas de éstos, como esquinas y centro, orientándose siempre hacia los accesos, especialmente a la parte central y esquinas exteriores de los mismos. Esta posición tuvo el objetivo de ubicar puntos específicos en el horizonte formado por los cerros demarcados por los accesos mencionados, y en el Recinto Sureste, mediante el efecto de luz y sombras, para una observación más precisa se utilizaron las esquinas exteriores de los accesos para proyectar líneas de sombra, que debían coincidir en las esquinas exteriores de los nichos al entrar la luz del sol en sus salidas o puestas. Esta observación podía realizarse viendo este efecto desde cualquier parte del interior del recinto usado, sin la necesidad de que el observador se orientara hacia el astro durante el fenómeno astronómico.

En el registro de ángulos de azimut y elevación en ambas estructuras se percibe cierto grado de precisión y sistematización al momento de su construcción y definición de sus funciones astronómicas (Tablas 2 y 3). Cabe destacar que en ambos casos se repiten de forma muy notoria y destacable los alineamientos asociados a equinoccios, pasajes del sol por el antizenit y zenit, lo que indicaría una mayor importancia de estos alineamientos en este tатри, además de estar conectadas ambas estructuras por el eje equinoccial que atraviesa las portadas de felinos tallados del sector II-B.

Utilizando los principales alineamientos solares en Huánuco Pampa, los incas contaron con los marcadores necesarios para controlar los 365 días del año. Las fechas calculadas para los alineamientos solares presentes en Huánuco Pampa (para el año 1530 según el Calendario Gregoriano) serían:

$\begin{array}{lll}\text { Solsticio } & (\mathrm{S}) & : 22 \text { de diciembre. } \\ \text { Zenit } & (\mathrm{Z}) & : 24 \text { de febrero. } \\ \text { Equinoccio } & (\mathrm{EQ}) & : 21 \text { de marzo. } \\ \text { Antizenit } & (\mathrm{AZ}) & : 16 \text { de abril. } \\ \text { Solsticio } & (\mathrm{S}) & : 22 \text { de junio. } \\ \text { Antizenit } & (\mathrm{AZ}) & : 28 \text { de agosto. } \\ \text { Equinoccio } & (\mathrm{EQ}) & : 23 \text { de septiembre. } \\ \text { Zenit } & (\mathrm{Z}): 19 \text { de octubre. }\end{array}$

Según el Calendario Gregoriano, las fechas del año en que ocurrieron los solsticios y equinoccios en la época inca son casi las mismas que las de la actualidad, tal como lo demuestra un análisis sobre variaciones de tiempo de solsticios y equinoccios desde el siglo XVI al año 1998, realizado por Meeus (2000b: 346-352).

\section{Marcadores astronómicos y diseño del tampu}

Según nuestro registro a nivel de asentamiento, creemos que para el trazado del tampu primero se

\begin{tabular}{|c|c|c|c|c|}
\hline \multicolumn{2}{|c|}{ Alineamiento } & \multirow[t]{2}{*}{ Azimut } & \multirow{2}{*}{$\begin{array}{l}\text { Elevación } \\
\text { Horizonte }\end{array}$} & \multirow{2}{*}{$\begin{array}{r}\text { Fenómeno } \\
\text { asociado }\end{array}$} \\
\hline De: & A: & & & \\
\hline Esquina exterior sur de n1 & Centro de AE3 & $114^{\circ} 54^{\prime} 33^{\prime \prime}$ & $04^{\circ} 39^{\prime} 27^{\prime \prime}$ & SSSD \\
\hline Esquina exterior norte de $\mathrm{n} 4$ & Esquina interior sur de AE3 & $66^{\circ} 53^{\prime} 03^{\prime \prime}$ & $03^{\circ} 53^{\prime} 10^{\prime \prime}$ & SSSJ \\
\hline Esquina exterior sur de $\mathrm{n} 2$ & Esquina interior norte de AE3 & $88^{\circ} 43^{\prime} 05^{\prime \prime}$ & $02^{\circ} 58^{\prime} 20^{\prime \prime}$ & SSEQ \\
\hline Centro interior de $\mathrm{n} 2$ & Centro de AE3 & $99^{\circ} 10^{\prime} 42^{\prime \prime}$ & $02^{\circ} 21^{\prime} 33^{\prime \prime}$ & SSPZ \\
\hline Centro interior de $\mathrm{n} 3$ & Centro de AE3 & $80^{\circ} 05^{\prime} 39^{\prime \prime}$ & $02^{\circ} 49^{\prime} 45^{\prime \prime}$ & SSPAZ \\
\hline Esquina exterior norte de $\mathrm{n} 4$ & Centro de AE3 & $60^{\circ} 17^{\prime} 56^{\prime \prime}$ & $03^{\circ} 40^{\prime} 26^{\prime \prime}$ & SLPEN \\
\hline Esquina exterior norte de $\mathrm{n} 1$ & Centro de AE3 & $119^{\circ} 52^{\prime} 31^{\prime \prime}$ & $05^{\circ} 18^{\prime} 02^{\prime \prime}$ & SLPES \\
\hline Centro de AW2 & Centro de AE2 & $80^{\circ} 05^{\prime} 39^{\prime \prime}$ & $02^{\circ} 40^{\prime} 55^{\prime \prime}$ & SSPAZ \\
\hline Centro de AE2 & Centro de AW2 & $260^{\circ} 05^{\prime} 39^{\prime \prime}$ & - & PSPZ \\
\hline Esquina exterior norte de AW2 & Esquina exterior sur de AE2 & $88^{\circ} 43^{\prime} 20^{\prime \prime}$ & $02^{\circ} 47^{\prime} 51^{\prime \prime}$ & SSEQ \\
\hline Esquina exterior sur de AE2 & Esquina exterior norte de AW2 & $268^{\circ} 43^{\prime} 20^{\prime \prime}$ & - & PSEQ \\
\hline
\end{tabular}

Tabla 3. Alineamientos astronómicos registrados en la estructura incawasi de Huánuco Pampa. 


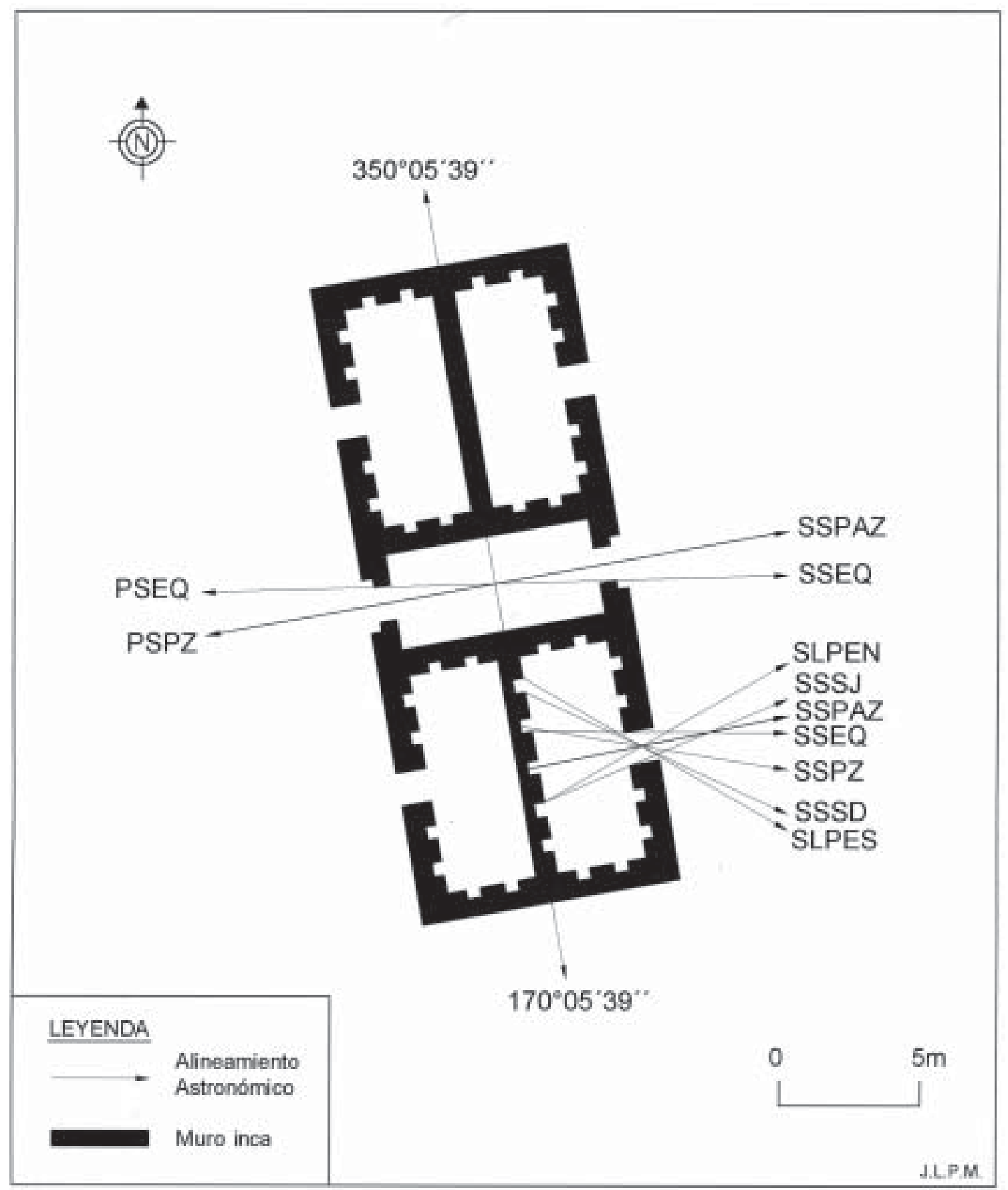

Figura 7. Alineamientos astronómicos en la estructura incawasi del Sector II B de Huánuco Pampa.

ubicó un punto central donde se construiría el ushnu. Este punto serviría para determinar los principales alineamientos astronómicos, fijándose como eje para el diseño de todo el asentamiento. En este diseño funcionarían como marcadores astronómicos los accesos y calles principales que dan a la plaza, y rasgos de la arquitectura de algunos conjuntos de estructuras. Diseños similares fueron observados en otros sitios incas, como Pumpu en Junín (Matos 1986) e Incawasi en Cañete (Hyslop 1985) y en otros tampus de la sierra central (ver Tabla 1). 
Cabe resaltar el lugar conocido como incamesanan al noroeste del asentamiento, donde se encuentran dos pequeñas plataformas, una a cada lado del camino inca que va hacia el norte. Entre estas dos plataformas pasa un alineamiento relacionado a la luna en su posición extremo norte. Estas plataformas sirvieron posiblemente como un referente astronómico observadas desde el ushnu. En Tikani (Isla del Sol, Bolivia) hay dos estructuras similares que servían de referentes astronómicos, observadas también desde una plataforma, pero relacionadas a la puesta del sol en el solsticio de junio (Dearborn et al. 1998: 250-252).

\section{Observatorios}

Proponemos que el ushnu y el incawasi funcionaron como observatorios astronómicos. Debido al complejo diseño del ushnu, creemos que posiblemente esta estructura fue usada para observaciones astronómicas directas, pues permite usar como marcadores astronómicos las tianas y los accesos. Las tianas habrían tenido una doble función: para que el observador se sentara y como marcador de referencia astronómica, sirviendo en el caso del sol como asientos simbólicos para este astro, tal como son descritas las "cillas" del sol en la crónica de Guamán Poma (1980 [1615]: 884 [898]) y la forma de usar estos asientos como lo refiere Francisco de Avila (cit. en Taylor 2000: 82).

En cambio, el diseño y la orientación de la estructura incawasi indican que sirvió para observaciones mediante efectos de luz y sombras, utilizando los accesos y nichos. Esta forma de observación se encuentra descrita en los documentos etnohistóricos (Guamán Poma 1980 [1615]: 884 [898]; Taylor 1999: 125), como también evidenciada en otras estructuras estudiadas en Ingapirca, Ecuador (Ziolkowski y Sadowski 1992: 48, 56-57), y Machu Picchu, Cusco (Dearborn et al. 1987: 347-350).

Fenómenos astronómicos como referentes de tiempo y espacio

Los alineamientos relacionados al sol, luna y estrellas registrados en este tampи permiten observar la forma de organización del "espacio" basándose en el "tiempo", y viceversa, usada por la sociedad incaica. Los fenómenos astronómicos de importancia, especialmente los más perceptibles a simple vista, sirvieron para organizar su calendario de actividades, a la vez que influyeron en la organización del espacio, orientando físicamente la arquitectura hacia los alineamientos asociados a estos fenómenos (Pino 2001 Ms-a). Este tipo de organización responde al pensamiento tradicional andino de concebir tiempo y espacio como una unidad, y usar un solo término para describirlo: Pacha. En el caso de Huánuco Pampa, fenómenos relacionados al sol y la luna fueron usados posiblemente con más énfasis en la organización del tiempo, indicando los alineamientos las fechas más importantes y las observaciones estelares para ubicar con más precisión el eje norte-sur y diseñar el asentamiento.

\section{Astronomía e ideología inca}

Un aspecto importante en Huánuco Pampa es la ornamentación de las estructuras en relación a los fenómenos astronómicos. Se observa que en las principales áreas donde se evidencian los alineamientos astronómicos están presentes figuras de felinos tallados en pares. Específicamente a ambos lados de los accesos consecutivos que forman un eje equinoccial en el sector II-B y a ambos lados de los dos accesos del ushnu relacionados a fenómenos estelares hacia el sur.

Las deidades principales de la sociedad inca fueron en su mayoría astros, por lo que casi todos los aspectos de su cosmovisión están relacionados a ellos y plasmados en sus asentamientos y objetos. La relación más importante de esta sociedad es con el sol, astro que tenían por ancestro. La verdadera imagen física de esta deidad elaborada por los incas era la de un niño, que siempre estaba acompañado por dos serpientes y dos felinos a los costados. Zuidema analizó esta verdadera imagen del sol, encontrando referencias en varias crónicas sobre un par felinos que lo acompañaban siempre (Zuidema 1974/76: 199-210). Asociada a esta concepción estaba la huaca de Susurpuquio, donde al Inka -algunas crónicas mencionan a Pachacuti y otras a Wiracocha-, el sol, bajo forma humana, se reveló en sueños mostrándole su verdadera imagen. Esta huaca según Zuidema (1974/76: 212-221) estaba relacionada al solsticio de junio. Además, según Guamán Poma en el Qoricancha, la imagen del sol y la ventana por donde este astro proyectaba su luz, estaba acompañada de dos felinos a cada lado y orientados hacia el sol: 
“(...) en el templo de Curi Cancha, que todas las paredes alto y bajo estaua uarnecida de oro finísimo y en lo alto del techo estaua colgado muchos cristales y a los dos lados dos leones apuntando el sol. Alumbraua de las ventanas la claridad de los dos partes, soplauan dos indios y se c... Entrauan el viento del soplo y salía un arco que ellos les llaman cuychi. $Y$ allí en medio se ponía el Ynga, hincado de rodillas, puesta las manos, el rrostro al sol y a la imagen del sol y decía su oración" (Guamán Poma 1980 [1615]: 262 [264]-263 [265]).

Según estas referencias, creemos que en Huánuco Pampa se trató de representar esta imagen del sol con los felinos a los costados, especialmente para la fecha del equinoccio cuando los primeros y últimos rayos atraviesan simultáneamente todas las portadas con felinos tallados, ubicadas en el Sector II-B. Este efecto visual sería usado por la sociedad inca de forma simbólica, posiblemente para mostrar su nexo filial con el sol, ante las poblaciones conquistadas.

Huánuco Pampa como síntesis del diseño inca de tampus

El registro de evidencias de alineamientos astronómicos en Huánuco Pampa, permite notar la influencia de la astronomía en el diseño de los tampus de este tipo. Existieron ciertas estructuras con funciones astronómicas para determinar posiciones de los astros en el horizonte en determinadas fechas, con lo cual se podía constituir un calendario, controlar el tiempo y organizar las actividades de diferente tipo. El ushnu y la estructura incawasi de Huánuco Pampa funcionaron como observatorios astronómicos, además de realizarse en ellas otras actividades. Evidencias similares fueron estudiadas en otras partes del Tawantinsuyu (Zuidema 1980; Ziolkowski y Sadowski 1992).

La evidencia material y arqueoastronómica presentada también confirma ciertas hipótesis propuestas para el Cusco, relacionadas al paso del sol por el zenit y el antizenit (Zuidema 1981: 322324), fenómenos astronómicos importantes en la agricultura, sobre todo en las fechas concernientes a las épocas de siembra y cosecha.

El ushnu de Huánuco Pampa, además de servir de centro de todas las orientaciones y divisiones espaciales del tampu, posee un diseño interno especial, relacionado con la mayoría de observaciones astronómicas (solares, lunares y estelares). Esto lo hace único en el Tawantinsuyu.

\section{Conclusiones}

Si bien el ushnu pudo haber sido originado de la simple idea abstracta del "lugar compuesto de piedras donde se filtra el agua", éste habría tenido un valor ceremonial en los rituales para conformarse como "el lugar donde se ofrendaban líquidos, o lugar de libaciones", función principal que continuó en la época inca, pero que a escala imperial la idea se fue sofisticando, adoptando el aspecto de plataformas, incluyendo tianas y pozos de ofrenda o cochas con sistemas de drenaje, adquiriendo un carácter más complejo, pero comenzando a conformar un nuevo concepto que quizá con el tiempo pudo haber sido redefinido. Además, fue adquiriendo una función muy importante en los territorios conquistados, que fue el de servir de eje para el diseño de las nuevas capitales provinciales a imagen del Cusco, por lo que fue necesario que se superponga la función de observatorio astronómico, con el que se pudo en algunos tampus organizar el espacio de forma radial y astronómica. Estos ushnus monumentales, pero a la vez artificiales en sí, trataron de ser entendidos según la imagen que los incas querían proyectar de un modo eficiente, al estar simbólicamente amarrados a sus deidades los astros y ser el punto de conexión entre los mundos de abajo (Uku Pacha) y de la superficie que habitaban (Kay Pacha) y demostrando en forma conjunta una "teatralidad del poder", en ceremonias donde el Inka o su representante ocupaba una posición central que conectaba todas las direcciones sagradas, no sólo de forma tridimensional, sino concibiendo a la vez dentro de esta abstracción la dimensión temporal. Elaborados de forma conjunta el ushnu y la organización del espacio crearon una conciencia colectiva uniforme, al tomar ideas que existían en los territorios conquistados y usarlas para legitimar el dominio ideológico del Imperio. Estos tampus, creaciones de nuevos Cuscos, conectaron simbólicamente los sitios sagrados de los territorios en conquista, mediante la ceremonia del Qapaq Ucha, a los sitios sagrados que los incas creaban, como el ushnu está conectado a todo el cosmos. 
Agradecimientos A Ramiro Matos, por su estimulo, consejos, asesoría en el desarrollo de la investigación y por la motivación por entender la cosmovisión andina. A Julian Idilio Santillana, por sus comentarios y valiosas clases de Arqueología Inca. A Manuel Perales, por sus sugerencias, comentarios y apoyo en toda la investigación. A Hernán Ramos, por su valiosa ayuda en el trabajo de campo y severa apreciación del manuscrito. A Martha Bell, Dawn Denoncourt y Adam Stack, por su invalorable ayuda en el trabajo de campo y registro de alineamientos en Huánuco Pampa. A Eberth Serrudo, por las conversaciones y las caminatas que realizamos a través del Qhapaqñan de Huánuco a Huari. A Gabriel Ramón, por sus comentarios, ayuda bibliográfica y permitirme viajar por la sierra del norte de Ancash y Trujillo. A Marcelo Saco, por su espontáneo apoyo a nuestro proyecto de investigación. A César Guerra, de manera muy especial, por su ayuda permanente y asesoría físico-matemática. Y a Norma Matos, por sus consejos y constante apoyo.

\section{REFERENCIAS CITADAS}

ALBORNOZ, C., 1967 [1582]. La instrucción para descubrir todas las guacas del Pirú y sus camayos y haziendas. Journal de la Société des Américanistes 56: 9-39.

AVENI, A., 2001. Skywatchers. A revised and updated version of skywatchers of Ancient Mexico. University of Texas Press, Austin.

BAUER, B. y D. DEARBORN, 1998. Astronomía e Imperio en los Andes. Centro de Estudios Regionales Andinos "Bartolomé de las Casas", Cusco.

CHAVEZ, M., 1970. Ciudades incas. Cusco Capital del Imperio. Wayka 3: 1-14.

D'ALTROY, T., 2003. The incas. Blackwell Publishing Ltd., Londres.

DEARBORN, D., K. SCHREIBER y R. WHITE, 1987. Intimachay: A december solstice observatory at Machu Picchu, Peru. American Antiquity 52 (2): 346-352.

DEARBORN, D., M. SEDDON y B. BAUER, 1998. The Sanctuary of Titicaca: Where the sun returns to earth. Latin American Antiquity 9 (3): 240-258.

DUVIOLS, P., 1976. La Capacocha, mecanismo y función del sacrificio humano, su proyección geométrica, su papel en la política integracionista y en la economía redistributiva del Tawantinsuyu. Allpanchis Phuturinqa 9: 11-57.

FARRINGTON, I., 1998. The concept of Cusco. Tawantinsuyu 5: 53-59.

GASPARINI, G. y L. MARGOLIES, 1977. Arquitectura inca. Centro de Investigaciones Históricas y Estéticas, Facultad de Arquitectura y Urbanismo, Universidad Central de Venezuela, Caracas.

GUAMAN POMA DE AYALA, F., 1980 [1615]. El primer nueva corónica y buen gobierno, J. Murra, R. Adorno y J. Urioste (Eds.). Editorial Siglo Veintiuno, México D. F.
HARTH-TERRE, E., 1964. El pueblo de Huánuco Viejo. Arquitecto Peruano 320-321: 1-20.

HERNANDEZ PRINCIPE, R., 1923. Mitología andina. Idolatría en Recuay. Revista Inca 1 (1).

HYSLOP, J., 1985. Incawasi, the New Cusco: Cañete, Lunahuaná, Perú. Institute of Andean Research, New York, BAR Series, Oxford.

-1990. Inca settlement planning. University of Texas Press, Austin.

MATOS, R., 1986. El ushnu de Pumpu. Cuicuilco 18: 45-61.

1994. Pumpu: Centro administrativo inca de la Puna de Junín. Editorial Horizonte, Lima.

-1995. Los incas de la sierra central del Perú. Revista de Arqueología Americana 8: 159-190.

MATOS, R., C. ARELLANO y D. BROWN, 1996. Asentamientos inca en Chakamarka y Tarmatambo (departamento de Junín). Problemas y criterios de interpretación para la reconstrucción de una provincia inca. I Encuentro Internacional de Peruanistas T I: 181-193. Universidad de Lima / Fondo de Cultura Económica, Lima.

MEDDENS, F., 1997. Function and meaning of the usnu in Late Horizon Peru. Tawantinsuyu 3: 5-14.

MEEUS, J., 2000a. Astronomical algorithms. Willmann-Bell, Inc. Richmond, Virginia.

2000b. Mathematical astronomy morsels. Willmann-Bell, Inc. Richmond, Virginia.

MORRIS, C., 1987. Arquitectura y estructura del espacio en Huánuco Pampa. Cuadernos 12: 27-45.

MORRIS, C. y D. THOMPSON, 1970. Huánuco Viejo: An inca administrative center. American Antiquity 35 (3): 344-362. 
1985. Huánuco Pampa: An inca city and its hinterland. Thames and Hudson, Londres.

PINO, J. L., 2001 Ms-a. El ushnu inca: Ritual, poder y orden astronómico en la sierra central del Chinchaysuyu. Ponencia presentada en XIII Congreso Peruano del Hombre y la Cultura Andina. Lima.

-2001 Ms-b. La idea de ushnu en los Andes Centrales. Ponencia presentada en XIII Congreso Peruano del Hombre y la Cultura Andina. Lima.

_2003 Ms. Observatorios y alineamientos astronómicos en el tampu inca de Huánuco Pampa, Perú. Ponencia presentada en el $51^{\circ}$ Congreso Internacional de Americanistas, Symposio ARQ-13, Santiago.

RAFFINO, R., D. GOBBO, R. VAZQUEZ, A. CAPPARELLI, V. MONTES, R. ITURRIZA, C. DESCHAMPS y M. MANNASERO, 1997. El ushnu de El Shincal de Quimivil. Tawantinsuyu 3: 22-39.

SERRUDO, E., 2002. El tambo real de Taparaku, HuánucoPerú. Arqueología y Sociedad 14: 123-139.

SHEA, D., 1966. El conjunto arquitectónico central en la plaza de Huánuco Viejo. Cuadernos de Investigación 1: 108116.

TAYLOR, G., 1999. Ritos y tradiciones de Huarochirí. Instituto Francés de Estudios Andinos e Instituto de Estudios Peruanos, Lima.

2000. Camac, camay y camasca, y otros ensayos sobre Huarochirí y Yauyos. Instituto Francés de Estudios Andinos, Lima.
URTON, G., 1985. La orientación en la astronomía quechua e inca. En La Tecnología en el Mundo Andino, H. Letchman y A. M. Soldi (Eds.), pp. 475-490. Universidad Nacional Autónoma de México, México D. F.

WACHTEL, N., 1973. Sociedad e ideología. Ensayos de historia y antropología andinas. Instituto de Estudios Peruanos, Lima.

ZIOLKOWSKI, M. y R. SADOWSKI, 1992. La arqueoastronomía en la investigación de las culturas andinas. Colección Pendoneros 9, Banco Central del Ecuador, Quito.

ZUIDEMA, T., 1974/76. La imagen del sol y la huaca de Susurpuquio en el sistema astronómico de los incas en el Cusco. Journal de la Société des Américanistes 63: 199-230.

1980. El ushnu. Revista de la Universidad Complutense 28 (117): 317-362.

-1981. Inca observations of the solar and lunar passages through Zenith and Anti-Zenith at Cusco. En Archaeoastronomy in the Americas, R. Williamson (Ed.), pp.319-342. Ballena Press. Los Altos, California.

-1983. Catachillay: El rol de Las Pleiades y de la Cruz del Sur y Alfa y Beta Centauri en el calendario de los incas. Fénix 28/29: 130-150.

-1989. Reyes y guerreros. Ensayos de cultura andina. Fomciencias, Lima.

-1995. El sistema de ceques del Cusco, la organización social de la capital de los incas. Fondo Editorial de la Pontificia Universidad Católica del Perú, Lima. 